\title{
Vibration tolerance of micro-electrodes
}

Kalhauge, Kristoffer Gram; Henrichsen, Henrik Hartmann; Wang, Fei; Hansen, Ole; Petersen, Dirch Hjorth

Published in:

Journal of Micromechanics and Microengineering

Link to article, DOI:

10.1088/1361-6439/aac58e

Publication date:

2018

Document Version

Early version, also known as pre-print

Link back to DTU Orbit

Citation (APA):

Kalhauge, K. G., Henrichsen, H. H., Wang, F., Hansen, O., \& Petersen, D. H. (2018). Vibration tolerance of micro-electrodes. Journal of Micromechanics and Microengineering, 28(9), [095010].

https://doi.org/10.1088/1361-6439/aac58e

\section{General rights}

Copyright and moral rights for the publications made accessible in the public portal are retained by the authors and/or other copyright owners and it is a condition of accessing publications that users recognise and abide by the legal requirements associated with these rights.

- Users may download and print one copy of any publication from the public portal for the purpose of private study or research.

- You may not further distribute the material or use it for any profit-making activity or commercial gain

- You may freely distribute the URL identifying the publication in the public portal

If you believe that this document breaches copyright please contact us providing details, and we will remove access to the work immediately and investigate your claim. 


\title{
Vibration tolerance of micro-electrodes
}

\author{
Kristoffer G Kalhauge ${ }^{1}$, Henrik H Henrichsen ${ }^{2}$, Fei Wang ${ }^{3}$, \\ Ole Hansen ${ }^{1}$ and Dirch H Petersen ${ }^{1}$ \\ ${ }^{1}$ Department of Micro and Nanotechnology, Technical University of Denmark, \\ DTU Ørsteds Plads Building 345C, DK-2800 Kgs Lyngby, Denmark \\ 2 CAPRES A/S, Scion-DTU, Building 373, DK-2800 Kgs Lyngby, Denmark \\ 3 Department of Electronic and Electrical Engineering, Southern University of \\ Science and Technology, Shenzhen, China 518055 \\ E-mail: kgka@nanotech.dtu.dk
}

\begin{abstract}
.
Micro four-point probe measurements can be used for extremely accurate electrical characterization of advanced electronic materials. The measurements are, however, sensitive to environmental vibrations which may compromise measurement quality severely. We have experimentally measured the vibration tolerance of two different micro-electrode designs (straight and three-way flexible) on two different samples (Indium-Tin-Oxide and highly doped Silicon). We show that three-way flexible electrodes are significantly more vibration tolerant (at least 50 times) compared to their straight counterparts. We have theoretically analyzed the vibration tolerance of the electrode designs and show excellent agreement between theory and experiment; as a result the theoretical framework may be used to predict vibration tolerance of any micro-electrode.
\end{abstract}

Submitted to: J. Micromech. Microeng. 


\section{Introduction}

For more than a century the four-point probe (4PP) has been a preferred method for precise electrical characterization of materials $\sqrt{1-3}$. However, the size of the probes and the contact force during engagement have limited its use for measurements on ultra thin materials [4,5].

Miniaturization obtained by use of microfabrication technologies has had a strong impact on micromanipulator performance [6] and also improved the performance of $4 \mathrm{PP}$ with the introduction of the micro four-point probe (M4PP) as an alternative to conventional 4PP [7]. During the past decade, M4PP measurements have become the most reliable method for electrical characterization of ultra-thin semiconducting layers 8. Today, fully automatic systems can perform sheet resistance [9] and micro Hall effect [10 11] measurements in scribe-line test pads using lock-in technique and configuration switching combined with advanced data treatment algorithms 12 for state-of-theart reliability [4,5, 13 .

An M4PP typically comprises (at least) four cantilever electrodes extending from a silicon probe body. The cantilever electrodes are silicon or silicon dioxide beams coated with metal (e.g., Ni). During measurements the contact force of each electrode is around 10-100 $\mu \mathrm{N}$, i.e., around five orders of magnitude lower than that of a conventional 4PP 8. M4PPs can be made of metal coated polymer 14, 15], or alternatively measurements may be done with independently movable probes 16 .

M4PP measurements rely on a static contact between the micro-electrodes and the test sample during the full duration of the data acquisition which lasts for several seconds. However, fully automatic metrology systems are most often placed in production environments with significant ambient vibrational noise, which could result in unintended motion of the contact points. If a static contact is not maintained during a measurement sequence, the result is discarded due to stringent measurement requirements imposed by the need for high reproducibility $[12,17,20]$.

The original M4PP electrodes were simple straight cantilevers (I-beams) [7] as shown in Figure 1a. Later, high aspect ratio, three-way flexible cantilever electrodes (L-beams, Figure 1b) were proposed for reducing abrasive wear of the electrode tips by forming a static contact 21,22.

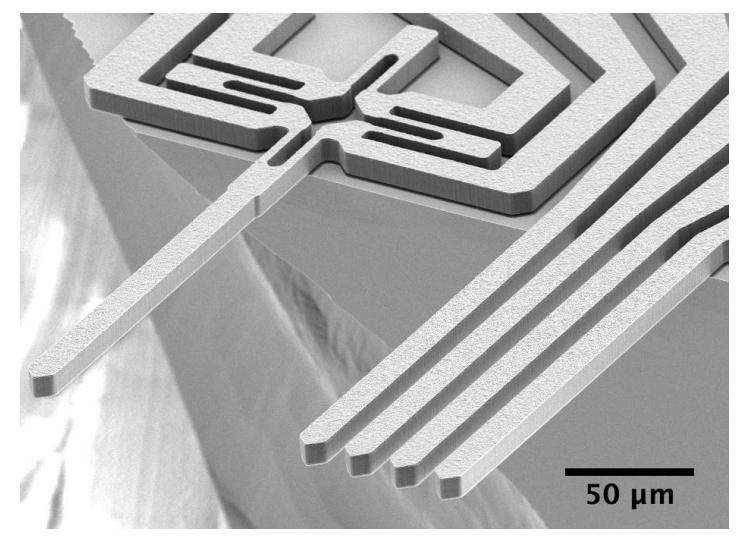

(a)

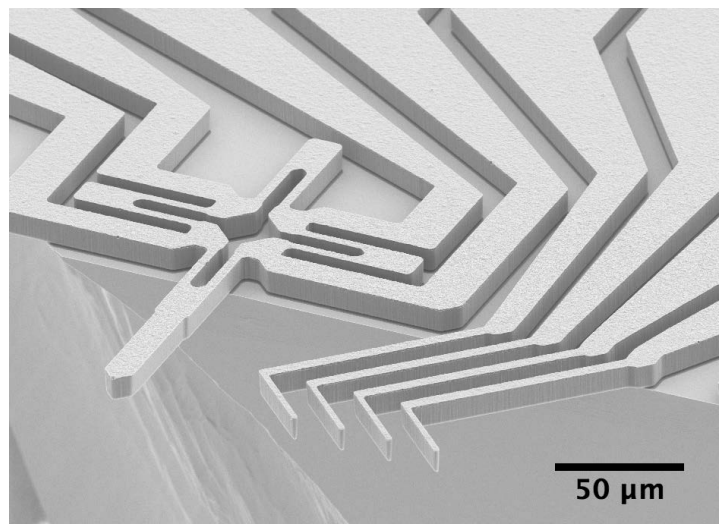

(b)

Figure 1: SEM images of micro four-point probes. (a) I-beam probes with straight electrodes. (b) L-beam probes with more flexible electrode beams. The images also show Wheatstone bridge strain-gauges (for surface detection) on both probes.

Here we experimentally determine the vibration tolerance of I-beam and L-beam cantilevers on two different sample surfaces as a function of engagement depth (i.e., contact force). We show experimentally that the L-beam has a higher vibration tolerance than that of the traditional I-beam. We also develop a theoretical model for the vibration tolerance of any cantilever with a known compliance tensor. We continue to calculate the vibration tolerance of the I-beam and L-beam cantilevers, and show that the model predictions are in excellent agreement with the experimental results. 


\section{Theory}

In a typical measurement instrument, the electrodes are engaged with the sample at a tilt angle $\phi$ (e.g., $\phi=$ $30^{\circ}$ ) and an engagement depth $\delta_{z}$ (e.g., $\left.\delta_{z}=500 \mathrm{~nm}\right)$ to provide sufficient contact force $F_{\mathrm{N}}$ for reliable electrical contact. The electrode-sample geometry is illustrated in Figure 2a for both I-beam and L-beam electrodes. Figure 2a also illustrates the sample based Cartesian coordinate system $(x, y, z)$ we shall use for analysis of vibration tolerance, while Figure $2 \mathrm{~b}$ shows the cantilever based coordinate system $\left(x^{\prime \prime}, y^{\prime \prime}, z^{\prime \prime}\right)$ which we shall use for analysis of the elastic behavior of the beams. Figure $2 \mathrm{~b}$ also defines the geometry of the L-beam, which is attached to the probe body at the hinge angle $\theta$ as illustrated in Figure 2a The analysis of vibration tolerance is simplified significantly if the electrode-sample contact is assumed to be point like such that the contact point can support forces, but not torques. Then the relation between the deflection vector $\boldsymbol{\delta}$ and force $\mathbf{F}$ on the electrode-tip is characterized by a stiffness $[\mathbf{K}]$ or compliance tensor $[\mathbf{C}]=[\mathbf{K}]^{-1}$, i.e., $\mathbf{F}=[\mathbf{K}] \boldsymbol{\delta}$ and $\boldsymbol{\delta}=[\mathbf{C}] \mathbf{F}$ as discussed by $\mathrm{F}$. Wang et al. 22 .

During electrode-sample engage two extreme cases may occur: Either static contact is obtained, i.e., $\boldsymbol{\delta}=$ $\delta_{z} \hat{\mathbf{z}}$ where $\hat{\mathbf{z}}$ is the unit vector normal to the surface of the sample. Or the cantilever slides on the surface until forces tangential to the surface vanish, i.e., $\mathbf{F}=$ $F_{\mathrm{N}} \hat{\mathbf{z}}=C_{z z}^{-1} \delta_{z} \hat{\mathbf{z}}$. In the analysis we shall assume sliding contact. During measurements, when the sample and probe are in contact, the sample may move relative to the probe body due to environmental noise vibrations, and then the contact point may or may not move on the sample depending on the vibration amplitude and properties of the contact and the electrode. We shall assume that the contact is maintained if the magnitude of the tangential force $F_{\mathrm{T}}$ is less than the total normal force $F_{\mathrm{N} \text {,tot }}$ times the friction coefficient $\mu$, i.e., $\left|F_{\mathrm{T}}\right| \leq \mu F_{\mathrm{N}, \text { tot }}$, otherwise the contact point will slide on the surface and compromise measurement quality.

Consider now in-plane sample-body vibrations $\tilde{\boldsymbol{\delta}}(t)$ with the amplitude $A$ at an arbitrary polar angle $\psi$ to the $x$-axis

$\tilde{\boldsymbol{\delta}}(t)=A \sin (\omega t)\left[\begin{array}{c}\cos \psi \\ \sin \psi \\ 0\end{array}\right]$,

where $\omega$ is the angular vibration frequency and $t$ is time. The resulting force due to engagement with sliding contact and added vibration is $\mathbf{F}=$ $F_{\mathrm{N}} \hat{\mathbf{z}}+[\mathbf{K}] \tilde{\boldsymbol{\delta}}(t)$ and thus the total normal force $F_{\mathrm{N}, \text { tot }}$ and tangential force $F_{\mathrm{T}}$ become

$F_{\mathrm{N}, \text { tot }}=C_{z z}^{-1} \delta_{z}+K_{\mathrm{N}}(\psi) A \sin (\omega t)$,

$F_{\mathrm{T}}=K_{\mathrm{T}}(\psi) A \sin (\omega t)$,

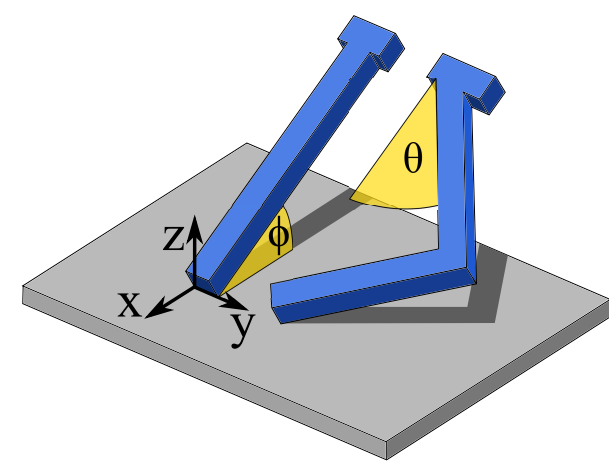

(a)

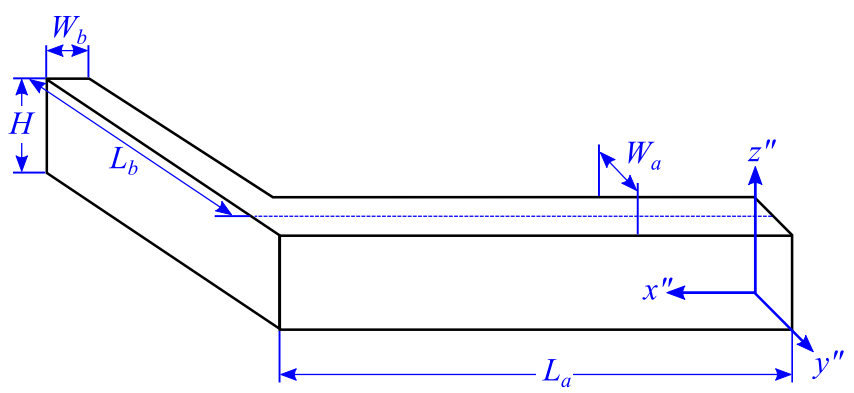

(b)

Figure 2: (a) Sketch of the electrode-sample geometry in the sample coordinate system. Both the I-beam and the L-beam electrodes are engaged with the sample at the tilt angle $\phi$. The L-beam is attached to the probe body with the hinge angle $\theta$. (b) Sketch of the L-beam electrode in the cantilever coordinate system illustrating the definition of geometry symbols.

where $K_{\mathrm{N}}(\psi)$ and $K_{\mathrm{T}}(\psi)$ are effective normal and transverse spring constants, respectively. In terms of the stiffness tensor elements the spring constants are

$K_{\mathrm{N}}(\psi)=K_{z x} \cos \psi+K_{z y} \sin \psi$,

$K_{\mathrm{T}}(\psi)=$

$\sqrt{\left(K_{x x} \cos \psi+K_{x y} \sin \psi\right)^{2}+\left(K_{y x} \cos \psi+K_{y y} \sin \psi\right)^{2}}$.

The worst case scenario occurs when $|\sin (\omega t)|=$ 1 , and since $K_{\mathrm{T}}(\psi)$ is always positive the vibration amplitude must fulfill

$$
\frac{A}{\delta_{z}} \leq \frac{\mu C_{z z}^{-1}}{K_{\mathrm{T}}(\psi)+\mu\left|K_{\mathrm{N}}(\psi)\right|} \equiv \Gamma(\psi)
$$

to avoid a sliding contact point according to the criterion $\left|F_{\mathrm{T}}\right| \leq \mu F_{\mathrm{N}, \text { tot }}$ discussed above. The vibration tolerance, in units of the engagement depth, for a vibration in the direction $\psi$ is thus $\Gamma(\psi)$.

Depending on the design of the electrodes two simplified cases can be identified, i.e., in case $K_{\mathrm{T}}(\psi) \gg \mu\left|K_{\mathrm{N}}(\psi)\right|$ the vibration tolerance $\Gamma(\psi) \simeq \mu /\left[C_{z z} K_{\mathrm{T}}(\psi)\right]$ is proportional to the 
friction coefficient, and in case $K_{\mathrm{T}}(\psi) \ll \mu\left|K_{\mathrm{N}}(\psi)\right|$ the vibration tolerance $\Gamma(\psi) \simeq 1 /\left[C_{z z}\left|K_{\mathrm{N}}(\psi)\right|\right]$ is independent on the friction coefficient.

The vibration tolerance for the I-beam is minimum at polar angles $\psi=0 \pm \pi$ and maximum at $\psi= \pm \frac{\pi}{2}$, and the same is roughly the case for the L-beam, albeit with a negligible offset. At the minimum and maximum the vibration tolerance becomes

$\Gamma(0)=\frac{\mu C_{z z}^{-1}}{\sqrt{K_{x x}^{2}+K_{y x}^{2}}+\mu\left|K_{z x}\right|}$,

$\Gamma\left(\frac{\pi}{2}\right)=\frac{\mu C_{z z}^{-1}}{\sqrt{K_{x y}^{2}+K_{y y}^{2}}+\mu\left|K_{z y}\right|}$,

respectively.

\subsection{Compliance tensors}

To proceed further, the compliance tensors of the cantilevers must be calculated and here we use Euler's beam equation 23 and the double-primed cantilever coordinate system of Figure 2b.

The calculation of the compliance tensor describing the I-beam, which is a prismatic beam of length $L_{\mathrm{I}}$, thickness $H_{\mathrm{I}}$ and width $W_{\mathrm{I}}$, proceeds by assuming that one end is rigidly clamped to the probe body. The resulting I-beam compliance tensor $\left[\mathbf{C}_{\mathrm{I}}^{\prime \prime}\right]$ in the cantilever coordinate system is

$\left[\mathbf{C}_{\mathrm{I}}^{\prime \prime}\right]=\left[\begin{array}{ccc}\frac{L_{\mathrm{I}}}{E W_{\mathrm{I}} H_{\mathrm{I}}} & 0 & 0 \\ 0 & \frac{4 L_{\mathrm{I}}^{3}}{E W_{\mathrm{I}}^{3} H_{\mathrm{I}}} & 0 \\ 0 & 0 & \frac{4 L_{\mathrm{I}}^{3}}{E W_{\mathrm{I}} H_{\mathrm{I}}^{3}}\end{array}\right]$

where $E=170 \mathrm{GPa}$ is Young's modulus of the beam material. Note, the element $C_{\mathrm{I}, x x}^{\prime \prime}$ is a low compliance element which is controlled by longitudinal deformation. In the remaining non-zero elements the effect of longitudinal deformation is insignificant and thus ignored.

The L-beam consists of two prismatic beams, the first beam (length $L_{\mathrm{a}}$, width $W_{\mathrm{a}}$, and height $H$ ) is clamped to the probe body, while the second beam (length $L_{\mathrm{b}}$, width $W_{\mathrm{b}}$, and height $H$ ) is connected to the first beam at a $90^{\circ}$ degree elbow. The compliance tensor $\left[\mathbf{C}_{\mathrm{L}}^{\prime \prime}\right]$ for the L-Beam cantilever was calculated by Wang et al. 22

$\left[\mathbf{C}_{\mathrm{L}}^{\prime \prime}\right]=$

$\frac{1}{E}\left[\begin{array}{ccc}\frac{12 L_{\mathrm{a}} L_{\mathrm{b}}^{2}}{W_{\mathrm{a}}^{3} H}+\frac{4 L_{\mathrm{b}}^{3}}{W_{\mathrm{b}}^{3} H} & -\frac{6 L_{\mathrm{a}}^{2} L_{\mathrm{b}}}{W_{\mathrm{a}}^{3} H} & 0 \\ -\frac{6 L_{\mathrm{a}} L_{\mathrm{b}}}{W_{\mathrm{a}}^{3} H} & \frac{4 L_{\mathrm{a}}^{3}}{W_{\mathrm{a}}^{3} H} & 0 \\ 0 & 0 & \frac{4 L_{\mathrm{a}}^{3}}{W_{\mathrm{a}} H^{3}}+\frac{4 L_{\mathrm{b}}^{3}}{W_{\mathrm{b}} H^{3}}+\frac{E L_{\mathrm{b}}^{2}}{k_{\tau}}\end{array}\right]$,

where $k_{\tau}$ is the torsion constant of the first prismatic bar of the L-beam $[24$

$k_{\tau}=\frac{E W_{\mathrm{a}}^{3} H}{2(1+\nu) L_{\mathrm{a}}}\left(\frac{1}{3}-\frac{64 W_{\mathrm{a}}}{\pi^{5} H} \beta\right)$,

\begin{tabular}{lrllr}
\hline \multicolumn{2}{c}{ L-beam } & & \multicolumn{2}{c}{ I-beam } \\
\cline { 1 - 2 } \cline { 5 - 5 } Symbol & $\begin{array}{r}\text { Value } \\
(\mu \mathrm{m})\end{array}$ & & Symbol & $\begin{array}{r}\text { Value } \\
(\mu \mathrm{m})\end{array}$ \\
\hline$H$ & 9.7 & & $H_{\mathrm{I}}$ & 9.9 \\
$L_{a}$ & 92.1 & $L_{\mathrm{I}}$ & 201.0 \\
$L_{b}$ & 36.9 & & \\
$W_{a}$ & 4.8 & & $W_{\mathrm{I}}$ & 12.0 \\
$W_{b}$ & 2.3 & & \\
\hline$\theta$ & $45^{\circ}$ & $\theta_{\mathrm{I}}$ & $0^{\circ}$ \\
$\phi$ & $30^{\circ}$ & $\phi_{\mathrm{I}}$ & $30^{\circ}$ \\
\hline
\end{tabular}

Table 1: Dimensions of the two cantilever designs used as defined in Figure 2 The electrode pitch of both probes is $20 \mu \mathrm{m}$.

where $\nu$ is Poisson's ratio and

$\beta=\sum_{n=1}^{\infty} \frac{1}{(2 n-1)^{5}} \tanh \frac{(2 n-1) \pi H}{2 W_{\mathrm{a}}}$.

These compliance tensors describe the deflection of the cantilever tips relative to the probe body in response to an applied force. However, the probe body may not be perfectly rigidly attached to the bulk of the instrument, and thus it is necessary to include also a system compliance $\left[\mathbf{C}_{\varepsilon}^{\prime \prime}\right]$ to fully describe the electrode-sample interaction. Since the impact of system compliance is significant only in low compliance directions of the cantilevers a system compliance tensor with only a single non-zero element is sufficient, i.e.,

$\left[\mathbf{C}_{\varepsilon}^{\prime \prime}\right]=\left[\begin{array}{ccc}\varepsilon & 0 & 0 \\ 0 & 0 & 0 \\ 0 & 0 & 0\end{array}\right]$,

with the non-zero element assigned the value $\varepsilon=0.2$ $\mathrm{mm} / \mathrm{N}$, which is an insignificant compliance compared to all compliance elements except $C_{\mathrm{I}, x x}^{\prime \prime}$, which is an order of magnitude smaller than $\varepsilon$. As a result the total compliance tensors are $\left[\mathbf{C}_{\mathrm{I}_{\varepsilon}}^{\prime \prime}\right]=\left[\mathbf{C}_{\mathrm{I}}^{\prime \prime}\right]+\left[\mathbf{C}_{\varepsilon}^{\prime \prime}\right]$ and $\left[\mathbf{C}_{\mathrm{L}_{\varepsilon}}^{\prime \prime}\right] \simeq\left[\mathbf{C}_{\mathrm{L}}^{\prime \prime}\right]$ for the I-beam and L-beam, respectively. The method used for determining the magnitude of $\varepsilon$ is described in section 4

For vibration tolerance calculations in the sample coordinate-system the compliance tensors are then rotated using the rotation matrix 22

$$
[\mathbf{T}]=\left[\begin{array}{ccc}
\cos \theta \cos \phi & -\sin \theta & -\cos \theta \sin \phi \\
\sin \theta \cos \phi & \cos \theta & -\sin \theta \sin \phi \\
\sin \phi & 0 & \cos \phi
\end{array}\right] .
$$

Table 1 shows the dimensions of the I-beam and L-beam cantilevers used in vibration tolerance calculations. The dimensions reported in Table 1 were measured on SEM images of the beams. For the calculations the elastic parameters, Young's modulus 


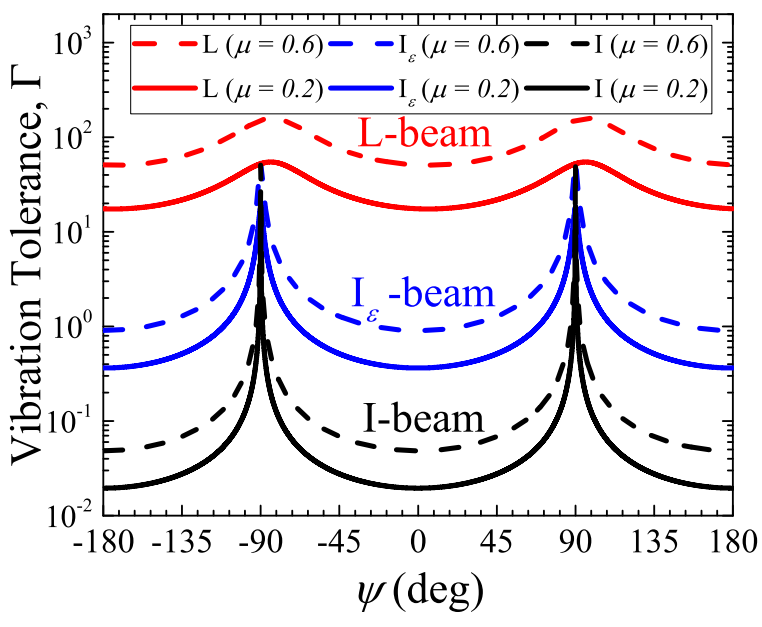

Figure 3: Calculated normalized vibration tolerance $\Gamma$ for I-beam (black) and L-beam (red) cantilevers as a function of the polar angle $\psi$ assuming friction coefficients $\mu=0.2$ (full) and 0.6 (dashed). The curves in blue, labeled $\mathrm{I}_{\varepsilon^{-}}$ beam, are calculated by taking into account the system compliance.

$E=170 \mathrm{GPa}$ and Poisson's ratio $\nu=0.22$, were used 25].

Figure 3 shows the calculated vibration tolerances for I-beam (black) and L-beam (red) electrodes as a function of the polar angle $\psi$ for two different values ( $\mu=0.2$ full lines and $\mu=0.6$ dashed lines) of the electrode-sample friction coefficient. The curves labelled $\mathrm{I}_{\varepsilon}$-beam (blue) results when the system compliance is included; the system compliance has a major effect on the calculated vibration tolerance for the I-beam as seen. The calculated vibration tolerance of the L-beam is roughly a factor of 50 higher than that of the $\mathrm{I}_{\varepsilon}$-beam.

\section{Experiment}

The vibration tolerance of the cantilever probes $\left(\mathrm{I}_{\varepsilon^{-}}\right.$ beam and L-beam) shown in Figure 1 was characterized experimentally by intentional vibration of the sample during dual configuration sheet resistance measurements. The sample to be measured was placed on a calibrated piezoelectric element, which was driven at a frequency of $100 \mathrm{~Hz}$ and with the vibration amplitude $A$ steadily increased during a measurement series. The direction of the sample vibration was aligned to the direction of minimum vibration tolerance for the cantilevers, i.e., $\psi \simeq 0$. Measurements were done on two different samples, i.e., on a silicon sample ( $\mathrm{Si})$ with a highly doped p-type shallow junction and on an indium-tin-oxide (ITO) sample with sheet resis- tances of $380 \Omega$ and $90 \Omega$, respectively. Sheet resistance measurements were done using an M200 tool from CAPRES A/S, and performed according to industry standards with a $1 \%$ median filter on the dual configuration sheet resistance measurements 26.

Measurement failure is interpreted as caused by motion of the cantilever contact points on the sample surface. Thus, the absolute vibration tolerance $\Gamma \delta_{z}$ was experimentally defined as the minimum vibration amplitude $A$ where the dual configuration sheet resistance measurement failed five times in a row (Note in clarification: at larger amplitudes resistance measurements almost always fail, while smaller amplitudes result in less frequent measurement failures.). In this fashion the vibration tolerance was measured as a function of the engagement depth $\delta_{z}$, which was varied in the range from 500 to $3000 \mathrm{~nm}$, with an estimated error of $200 \mathrm{~nm}$, as controlled by the probe stage on the M200. The engagement depth is measured as the set travel of the probe stage beyond detection of the surface. Representative measurements of the vibration tolerances of the $\mathrm{I}_{\varepsilon}$-beams and the $\mathrm{L}$ beams on the two samples are reported in Figure 4

\section{Results and discussion}

The experimental vibration tolerance data in Figure 4 show that, indeed, the absolute vibration tolerance increases approximately linearly with increased engagement depth $\delta_{z}$ for each combination of probe (I-beam or L-beam) and sample (Si or ITO) as expected. The vibration tolerance of the L-beam is much larger than that of the $\mathrm{I}_{\varepsilon}$-beam, and is, within measurement error, independent of the properties of the sample. This behavior is in stark contrast to the vibration tolerance of the $\mathrm{I}_{\varepsilon}$-beam, which is strongly dependent of the properties of the sample, i.e., the vibration tolerance on $\mathrm{Si}$ is an order of magnitude lower than that on ITO. This sample dependence of the vibration tolerance can be understood by inspecting post-measurement SEM images (not shown) of the sample surfaces, which show rather deep indentations from the $\mathrm{I}_{\varepsilon}$-beam on the ITO sample, but not on the $\mathrm{Si}$ sample. The indentations on the ITO sample effectively prevents the normal sliding motion on the surface; thereby the vibration tolerance is increased significantly.

In Figure 4 error bars are not reported, but there are sources of error on both axes. The larger error is that of the engagement depth, which is determined based on a threshold signal from the built-in strain gauge on the probes (Figure 1) and the motion of the calibrated probe stage of the M200 tool beyond this threshold. The threshold detection is expected to give a systematic error of at most $200 \mathrm{~nm}$. The probe stage has a resolution of $2.5 \mathrm{~nm}$ and a position stability of 


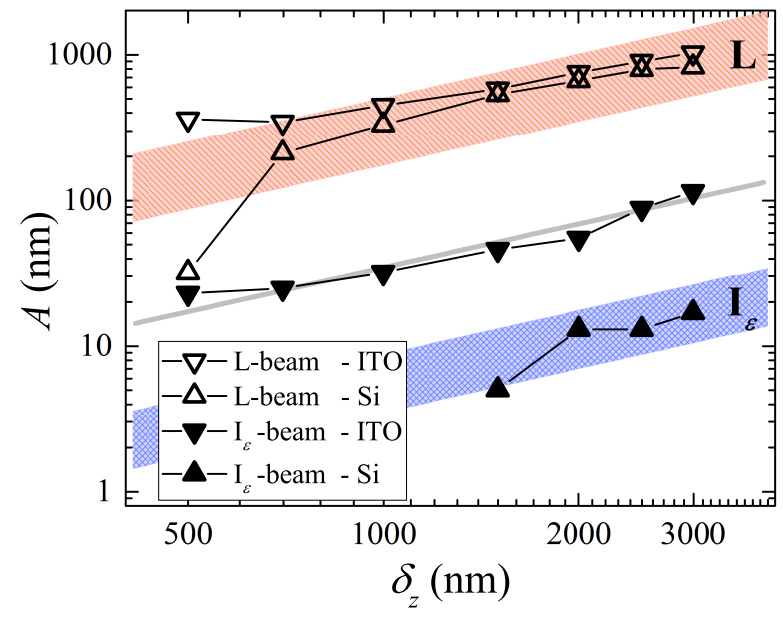

Figure 4: Experimental absolute vibration tolerance $A$ as function of engagement depth $\delta_{z}$ for $\mathrm{I}_{\varepsilon}$-beam and L-beam electrodes on $\mathrm{Si}$ and ITO samples. Theoretical predictions of the vibration amplitude for $\mathrm{I}_{\varepsilon}$-beam and L-beam electrodes are shown as the blue and red bands respectively, for friction coefficients in the range $\mu=0.2$ to 0.6. The full grey line is the theoretical prediction for the $\mathrm{I}_{\varepsilon}$-beam with an artificially large friction coefficient $(\mu \rightarrow \infty)$, which agrees well with $\mathrm{I}_{\varepsilon}$-beam measurements on ITO.

$10 \mathrm{~nm}$ peak-to-peak; thus it contributes with a random error of at most $5.6 \mathrm{~nm}$. The vibration amplitude is much more accurately determined, and here the main source of error is environmental vibration noise (a few $\mathrm{nm}$ ) during the measurements. This noise affected the measurements for the $\mathrm{I}_{\varepsilon}$-beam on $\mathrm{Si}$ where data could not be obtained at low engagement depths (below 1500 $\mathrm{nm})$.

The experimental vibration tolerance of the Lbeam is in very good agreement with the calculated vibration tolerance as illustrated by the red band (top) in Figure 4, which shows calculated vibration tolerance for friction coefficients in the range from $\mu=0.2$ to 0.6 ; i.e., almost all measurement points for both samples are within this band. The blue band (bottom) in Figure 4 illustrates a similar calculation for the $\mathrm{I}_{\varepsilon}$-beam assuming a system compliance $\varepsilon=0.2 \mathrm{~mm} / \mathrm{N}$, and again the agreement between experiment and theory is very good and essentially all measurement points on $\mathrm{Si}$ are within the band defined by the friction coefficient range $(0.2$ to 0.6$)$.

The vibration tolerance of the $\mathrm{I}_{\varepsilon}$-beam on ITO is an order of magnitude higher than that on $\mathrm{Si}$ and the theoretical prediction (blue band in Figure
4), but as mentioned above the indentation of the electrode in the sample prevents the usual sliding motion on the surface. We may model this behavior by allowing an artificially high friction coefficient (i.e., $\mu \rightarrow \infty)$ and then the normalized vibration tolerance becomes $\Gamma(0) \simeq 1 /\left(C_{z z}\left|K_{z x}\right|\right)$, which is independent on $\mu$. By fitting this simplified expression to the measurement data for the $\mathrm{I}_{\varepsilon}$-beam on ITO using the system compliance $\varepsilon$ as the fitting parameter produces the full grey line in Figure 4 for $\varepsilon=0.2 \mathrm{~mm} / \mathrm{N}$, and the agreement with measurements is excellent. Thus, this experiment essentially measures the system compliance, and the resulting system compliance was also used to explain the data for the $\mathrm{I}_{\varepsilon}$-beam on $\mathrm{Si}$ experiments in the previous paragraph.

\section{Conclusion}

We have measured the vibration tolerance of Ibeam and L-beam electrodes on ITO and Si samples. The measurements show that L-beam electrodes are at least 50 times more vibration tolerant than Ibeam electrodes. We have explained this behavior theoretically and the model of vibration tolerance we have developed is in excellent agreement with the experiments. Our theoretical framework may thus serve as a valuable tool for future micro-electrode design focusing on improved vibration tolerance.

\section{Acknowledgments}

The authors are thankful for financial support from H2020 European Project Nos. 692527 and 688225 . We would further like to thank Lior Shiv from Capres A/S for fruitful discussions on cantilever design.

\section{References}

[1] L. B. Valdes. Resistivity Measurements on Germanium for Transistors. Proceedings of the IRE, 42(2):420-427, 1954.

[2] F. M. Smits. Measurement of Sheet Resistivities with the Four-Point Probe. Bell System Technical Journal, 37(3):711-718, 1958

[3] I. Miccoli, F. Edler, H. Pfnür, and C. Tegenkamp. The 100th anniversary of the four-point probe technique: the role of the probe geometries in isotropic and anisotropic systems. Journal of Physics: Condensed Matter, 27(22), 2015.

[4] T. H. Clarysse, A. Moussa, F. Leys, R. Loo, W. Vandervorst, M. C. Benjamin, R. J. Hillard, V. N. Faifer, M. I. Current, R. Lin, and D. H. Petersen. Accurate Sheet Resistance Measurement on Ultra-Shallow Profiles. MRS Proceedings, 912:1-7, 2006.

[5] T. Clarysse, A. Moussa, B. Parmentier, J. Bogdanowicz, W. Vandervorst, H. Bender, M. Pfeffer, M. Schellenberger, P. F. Nielsen, S. Thorsteinsson, R. Lin, and D. H. Petersen. Photovoltage versus microprobe sheet resistance measurements on ultrashallow structures. Journal of Vacuum Science \& Technology B, Nanotechnology and 
Microelectronics: Materials, Processing, Measurement, and Phenomena, 28(1):C1C8-C1C14, 2010.

[6] S. Mekid, S. Bashmal, and H. M. Ouakad. Nanoscale Manipulators: Review of Conceptual Designs Through Recent Patents. Recent Patents on Nanotechnology, 10(1):44-58, 2016.

[7] C. L. Petersen, T. M. Hansen, P. Bøggild, A. Boisen, O. Hansen, T. Hassenkam, and F. Grey. Scanning microscopic four-point conductivity probes. Sensors and Actuators, A: Physical, 96(1):53-58, 2002.

[8] D. H. Petersen, O. Hansen, T. M. Hansen, P. Bøggild, R. Lin, D. Kjær, P. F. Nielsen, T. Clarysse, W. Vandervorst, E. Rosseel, N. S. Bennett, and N. E. B. Cowern. Review of electrical characterization of ultrashallow junctions with micro four-point probes. Journal of Vacuum Science 8 Technology B: Microelectronics and Nanometer Structures, 28(1):C1C27, 2010.

[9] S. Thorsteinsson, F. Wang, D. H. Petersen, T. M. Hansen, D. Kjær, R. Lin, J. Y. Kim, P. F. Nielsen, and O. Hansen. Accurate microfour-point probe sheet resistance measurements on small samples. Review of Scientific Instruments, 80(5), 2009.

[10] D. H. Petersen, O. Hansen, R. Lin, and P. F. Nielsen. Micro-four-point probe Hall effect measurement method. Journal of Applied Physics, 104(1), 2008.

[11] D. C. Worledge and P. L. Trouilloud. Magnetoresistance measurement of unpatterned magnetic tunnel junction wafers by current-in-plane tunneling. Applied Physics Letters, 83(1):84-86, 2003.

[12] D. H. Petersen. Micro Four-Point Probe and Micro Hall Effect. Phd thesis, Technical University of Denmark, 2009.

[13] T. Clarysse, J. Bogdanowicz, J. Goossens, A. Moussa, E. Rosseel, W. Vandervorst, D. H. Petersen, R. Lin, P. F. Nielsen, O. Hansen, G. Merklin, N. S. Bennett, and N. E. B. Cowern. On the analysis of the activation mechanisms of sub-melt laser anneals. Materials Science and Engineering B: Solid-State Materials for Advanced Technology, 154-155(1-3):24-30, 2008.

[14] J. K. Kim, Y. S. Choi, and D. W. Lee. Surface-adaptable all-metal micro-four-point probe with unique configuration. Journal of Micromechanics and Microengineering, 25(7), 2015

[15] S. Keller, S. Mouaziz, G. Boero, and J. Brugger. Microscopic four-point probe based on SU-8 cantilevers. Review of Scientific Instruments

[16] S. B. Kjeldby, O. M. Evenstad, S. P. Cooli, and J. W. Wells. Probing the dimensionality using a simplified 4-probe method. Journal of Physics: Condensed Matter, 29(39), 2017

[17] H. H. Henrichsen, O. Hansen, D. Kjær, P. F. Nielsen, F. Wang, and D. H. Petersen. Precision of single-engage micro Hall effect measurements. 2014 International Workshop on Junction Technology, IWJT 2014, pages $55-58,2014$.

[18] D. C. Worledge. Reduction of positional errors in a fourpoint probe resistance measurement. Applied Physics Letters, 84(10):1695-1697, 2004.

[19] D. Kjaer, O. Hansen, F. W. Østerberg, H. H. Henrichsen, C. Markvardsen, P. F. Nielsen, and D. H. Petersen. Characterization of positional errors and their influence on micro four-point probe measurements on a $100 \mathrm{~nm}$ $\mathrm{Ru}$ film. Measurement Science and Technology, 26(9), 2015 .

[20] A. Cagliani, F. W. Østerberg, O. Hansen, L. Shiv, P. F. Nielsen, and D. H. Petersen. Breakthrough in currentin-plane tunneling measurement precision by application of multi-variable fitting algorithm. Review of Scientific Instruments, 88(9), 2017.

[21] D. H. Petersen, O. Hansen, T. M. Hansen, P. R. E. Petersen, and P. Bøggild. Static contact micro four-point probes with $<11 \mathrm{~nm}$ positioning repeatability. Microelectronic Engineering, 85(5-6):1092-1095, 2008.

[22] F. Wang, D. H. Petersen, H. V. Jensen, C. Hansen, D. Mortensen, L. Friis, and O. Hansen. Three-way flexible cantilever probes for static contact. Journal of Micromechanics and Microengineering, 21:085003, 2011.

[23] James Monroe Gere. Mechanics of Materials. Thomson Learning, ISBN 0-534-41793-0, sixth edition, 2008.

[24] S. Timoshenko and J. N. Goodier. Theory of Elasticity. McGraw-Hill, ISBN 0070701229, third edition, 1970.

[25] W. N. Sharpe, B. Yuan, R. Vaidyanathan, and R. L. Edwards. Measurements of Young 'S Modulus , Poisson' S Ratio, and Tensile Strength of Polysilicon. Proceedings of the Tenth IEEE International Workshop on Microelectromechanical Systems, 429:424-429, 1997.

[26] CAPRES A/S homepage. http://www.capres.com/ Accessed: 18-12-2017. 